\title{
SYMMETRY IN THE VANISHING OF EXT OVER GORENSTEIN RINGS
}

\author{
CRAIG HUNEKE and DAVID A. JORGENSEN*
}

\begin{abstract}
We investigate symmetry in the vanishing of Ext for finitely generated modules over local Gorenstein rings. In particular, we define a class of local Gorenstein rings, which we call $\mathrm{AB}$ rings, and show that for finitely generated modules $M$ and $N$ over an $\mathrm{AB}$ ring $R, \operatorname{Ext}_{R}^{i}(M, N)=0$ for all $i \gg 0$ if and only if $\operatorname{Ext}_{R}^{i}(N, M)=0$ for all $i \gg 0$.
\end{abstract}

\section{Introduction}

Let $R$ be a local Gorenstein ring and let $M$ and $N$ denote finitely generated $R$-modules. This paper is concerned with the relation between the vanishing of all higher $\operatorname{Ext}_{R}(M, N)$ and the vanishing of all higher $\operatorname{Ext}_{R}(N, M)$. As a means of investigation we concentrate on the more natural duality between the vanishing of all higher $\operatorname{Ext}_{R}(M, N)$ and the vanishing of all higher Tor modules where either $M$ or $N$ is replaced by its dual $M^{*}\left(:=\operatorname{Hom}_{R}(M, R)\right)$ or $N^{*}$.

Our interest in this topic came about in part from the following striking result proved recently by Avramov and Buchweitz [2, Thm. III]. Suppose $M$ and $N$ are finitely generated modules over a complete intersection $R$. Then the following are equivalent:

(1) $\operatorname{Tor}_{i}^{R}(M, N)=0$ for all $i \gg 0$

(2) $\operatorname{Ext}_{R}^{i}(M, N)=0$ for all $i \gg 0$

(3) $\operatorname{Ext}_{R}^{i}(N, M)=0$ for all $i \gg 0$.

Their proof relies heavily on the use of certain affine algebraic sets associated to $M$ and $N$, called support varieties. In their paper [2], Avramov and Buchweitz raise the question of what class of rings satisfy these equivalences for all finitely generated modules $M$ and $N$. They point out this class lies somewhere between

\footnotetext{
* The first author was partially supported by the NSF and the second author was partially supported by the NSA. This work was done while the second author was visiting Kansas University. He thanks KU for their generous support.

Received October 1, 2001; in revised form June 25, 2002.
} 
complete intersections and local Gorenstein rings, but mention that they do not know whether this class is equal to either complete intersections or Gorenstein rings. In this paper we introduce a class of local Gorenstein rings, which we call $A B$ rings, and prove that $\mathrm{AB}$ rings satisfy the property that for all finitely generated modules $M$ and $N$, the following are equivalent (Theorem 4.1):

(1) $\operatorname{Ext}_{R}^{i}(M, N)=0$ for all $i \gg 0$

(2) $\operatorname{Ext}_{R}^{i}(N, M)=0$ for all $i \gg 0$.

Regular local rings are $\mathrm{AB}$ rings, and if $R$ is an $\mathrm{AB}$ ring, then $R /\left(x_{1}, \ldots, x_{c}\right)$ is also an $\mathrm{AB}$ ring whenever $x_{1}, \ldots, x_{c}$ is a regular sequence (see Proposition 3.3). This implies that complete intersections are $\mathrm{AB}$ rings. Even when restricted to the case of a complete intersection, our proof of the above equivalence avoids the use of support varieties, and in some ways is more direct than the methods of [2]. We also prove that local Gorenstein rings of minimal possible multiplicity are $\mathrm{AB}$ rings, for the strong reason that over such rings (except when the embedding dimension is 2) all large $\operatorname{Ext}_{R}(M, N)$ vanish if and only if either $M$ or $N$ has finite projective dimension. See Theorem 3.6 for a precise statement. These rings are not complete intersections in general, so that in particular the class of $\mathrm{AB}$ rings is strictly larger than that of complete intersections. In Theorem 3.8 and Proposition 4.4 we show that there also exist $\mathrm{AB}$ rings $R$ which are not complete intersections and over which there are finitely generated modules $M$ and $N$ such that $\operatorname{Ext}_{R}^{i}(M, N)=0$ for all $i>\operatorname{dim} R$ even though both $M$ and $N$ have infinite projective dimension over $R$.

$\mathrm{An} \mathrm{AB}$ ring $R$ is a local Gorenstein ring defined by the property that there is a constant $C$, depending only on the ring, such that if $\operatorname{Ext}_{R}^{i}(M, N)=0$ for all $i \gg 0$, then $\operatorname{Ext}_{R}^{i}(M, N)=0$ for all $i>C$. As far as we know every Gorenstein ring is an $\mathrm{AB}$ ring; we have been unable to find an example which is not. The name 'AB' stands for both Auslander-Bridger and AvramovBuchweitz.

The paper is organized as follows. In Section 1 we give some preliminary and straightforward results concerning the relationship of Ext and Tor. In Section 2 we prove a basic result concerning what holds over an arbitrary local Gorenstein ring. Specifically, if $M$ and $N$ are finitely generated maximal Cohen-Macaulay modules over a local Gorenstein ring $R$, then the following are equivalent:

(1) $\operatorname{Tor}_{i}^{R}(M, N)=0$ for all $i \gg 0$,

(2) $\operatorname{Ext}_{R}^{i}\left(M, N^{*}\right)=0$ for all $i \gg 0$, and

(3) $\operatorname{Ext}_{R}^{i}\left(N, M^{*}\right)=0$ for all $i \gg 0$. 
Section 3 introduces $\mathrm{AB}$ rings, details their basic properties, and gives the main examples. In Section 4 we prove the main theorem of symmetry in the vanishing of Ext over AB rings. Section 5 contains some independent observations concerning what the vanishing of Ext means. In particular we relate the vanishing of $d$ consecutive Ext modules ( $d$ being the dimension of the ring) to the Cohen-Macaulay property of a related tensor product. We include some questions in a final section.

\section{Preliminaries}

In this section we set notation and discuss some basic facts which will be used throughout the paper.

Unless otherwise stated, we will assume $R$ to be a local Gorenstein ring. Also, $M$ and $N$ will denote finitely generated $R$-modules. For an $R$-module $M$ we let $M^{*}$ denote its dual $\operatorname{Hom}_{R}(M, R)$. If $M$ is maximal Cohen-Macaulay then it is also reflexive, meaning $M^{* *} \simeq M$ (assuming $R$ is Gorenstein).

By a complete intersection we mean a local ring whose completion with respect to the maximal ideal is the quotient of a regular local ring by a regular sequence.

For a local ring $R$, we let embdim $R$ denote its embedding dimension.

\section{Syzygies and Conversions for Ext and Tor}

Suppose $M$ is an $R$-module. Then for $i \geq 0$ we let $M_{i}$ denote image $f_{i}$, where $f_{i}$ is the $i$ th differential in a minimal free resolution

$$
\mathbf{F}: \quad \cdots \rightarrow F_{2} \stackrel{f_{2}}{\longrightarrow} F_{1} \stackrel{f_{1}}{\longrightarrow} F_{0} \stackrel{f_{0}}{\longrightarrow} M \rightarrow 0
$$

of $M$. These $M_{i}$ are the non-negative syzygies of $M$. They are unique up to isomorphism, or if one considers a non-minimal resolution any two are stably isomorphic.

Now suppose that $M$ is a maximal Cohen-Macaulay $R$-module. Let

$$
\text { G: } \quad \cdots \rightarrow G_{2} \stackrel{g_{2}}{\longrightarrow} G_{1} \stackrel{g_{1}}{\longrightarrow} G_{0} \rightarrow M^{*} \rightarrow 0
$$

a minimal free resolution of its dual $M^{*}$. Since $M^{*}$ is maximal Cohen-Macaulay, the dual sequence

$$
\mathbf{G}^{*}: \quad 0 \rightarrow M^{* *} \rightarrow G_{0}^{*} \stackrel{g_{1}^{*}}{\longrightarrow} G_{1}^{*} \stackrel{g_{2}^{*}}{\longrightarrow} G_{2}^{*} \rightarrow \cdots
$$

is exact. Using the fact that $M$ is reflexive, we can splice $\mathbf{F}$ and $\mathbf{G}^{*}$ together, getting the doubly infinite long exact sequence

$$
\mathrm{C}(M): \quad \cdots \rightarrow F_{2} \stackrel{f_{2}}{\longrightarrow} F_{1} \stackrel{f_{1}}{\longrightarrow} \underset{0}{F_{0}} \rightarrow \underset{-1}{G_{0}^{*}} \stackrel{g_{1}^{*}}{\longrightarrow} \underset{-2}{G_{1}^{*}} \stackrel{g_{2}^{*}}{\longrightarrow} G_{-3}^{*} \rightarrow \cdots .
$$


(Note the degree convention.) For $i \leq-1$ we set $M_{i}:=\operatorname{image}\left(g_{-i}^{*}\right)$. These are the negative syzygies of $M$. They are unique up to isomorphism. Note that $M_{i}$ is again maximal Cohen-Macaulay for all $i$ when $M$ is.

We now list some properties of the long exact sequences $\mathrm{C}(M)$.

Lemma 1.1. Let $M$ be a finitely generated maximal Cohen-Macaulay $R$ module, and let $N$ be a finitely generated $R$-module.

(1) $\mathrm{C}(M)^{*} \simeq \mathrm{C}\left(M^{*}\right)$

(2) $\left(M_{i}\right)^{*} \simeq\left(M^{*}\right)_{-i}$ for all $i$.

(3) $\operatorname{Tor}_{i}^{R}(M, N) \simeq \mathrm{H}_{i}(\mathrm{C}(M) \otimes N)$ for $i \geq 1$.

(4) $\operatorname{Ext}_{R}^{i}(M, N) \simeq \mathrm{H}_{-i-1}\left(\mathrm{C}(M)^{*} \otimes N\right)$ for $i \geq 1$.

(5) For fixed $t \geq 3$ and for $1 \leq i \leq t-2$ we have

(i) $\operatorname{Ext}_{R}^{i}\left(M_{-t}, N\right) \simeq \operatorname{Tor}_{t-i-1}^{R}\left(M^{*}, N\right)$

and

(ii) $\operatorname{Tor}_{i}^{R}\left(M_{-t}, N\right) \simeq \operatorname{Ext}_{R}^{t-i-1}\left(M^{*}, N\right)$.

Proof. Conditions (1)-(3) are straightforward. Condition (5) follows easily from (1)-(4), so only Condition (4) needs some explanation. The critical fact we need to show is that for any complex of free $R$-modules $\mathbf{F}, \operatorname{Hom}_{R}(\mathbf{F}, N)$ and $\operatorname{Hom}_{R}(\mathbf{F}, R) \otimes_{R} N$ are isomorphic as complexes: write

$$
\mathbf{F}: \quad \cdots \rightarrow F_{i+1} \stackrel{f_{i+1}}{\longrightarrow} F_{i} \stackrel{f_{i}}{\longrightarrow} F_{i-1} \rightarrow \cdots,
$$

where the $F_{i}$ are free $R$-modules. The natural maps $h_{i}: \operatorname{Hom}_{R}\left(F_{i}, R\right) \otimes_{R} N \rightarrow$ $\operatorname{Hom}_{R}\left(F_{i}, N\right)$ given by $f \otimes n \mapsto\{a \mapsto f(a) n\}$ are isomorphisms since $F_{i}$ is free. It is easy to check that the diagram

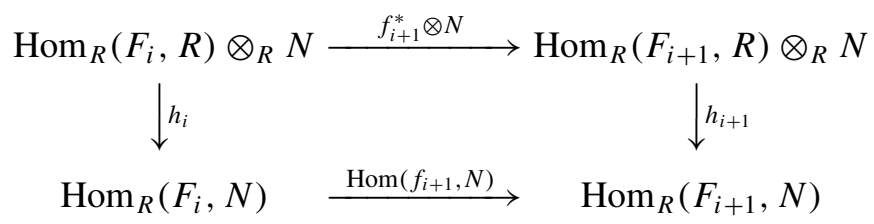

is commutative, and this establishes our fact.

Suppose $M$ and $N$ are $R$-modules with $M$ maximal Cohen-Macaulay. Then $\operatorname{Ext}^{i}(M, R)=0$ for all $i \geq 1$, and so by shifting along the short exact sequences $0 \rightarrow N_{n+1} \rightarrow G_{n} \rightarrow N_{n} \rightarrow 0$ (with $G_{n}$ free) we obtain isomorphisms

$$
\operatorname{Ext}_{R}^{i}(M, N) \simeq \operatorname{Ext}_{R}^{i+n}\left(M, N_{n}\right)
$$

for $i \geq 1$ and $n \geq 0$. 
The Change of Rings Long Exact Sequences of Ext and Tor

Suppose that $S$ is a commutative ring, $x$ is a non-zerodivisor of $S$ and $R:=$ $S /(x)$. Let $M$ and $N$ be $R$-modules. Then we have the change of rings long exact sequence of Ext [6, 11.65]

$$
\begin{aligned}
& \operatorname{Ext}_{R}^{1}(M, N) \leftarrow \operatorname{Ext}_{S}^{2}(M, N) \leftarrow \operatorname{Ext}_{R}^{2}(M, N) \leftarrow \\
& \operatorname{Ext}_{R}^{0}(M, N) \leftarrow \operatorname{Ext}_{S}^{1}(M, N) \leftarrow \operatorname{Ext}_{R}^{1}(M, N) \leftarrow 0,
\end{aligned}
$$

and the change of rings long exact sequence of Tor $[6,11.64]$

$$
\begin{aligned}
& \operatorname{Tor}_{1}^{R}(M, N) \rightarrow \operatorname{Tor}_{2}^{S}(M, N) \rightarrow \operatorname{Tor}_{2}^{R}(M, N) \rightarrow \\
& \operatorname{Tor}_{0}^{R}(M, N) \rightarrow \operatorname{Tor}_{1}^{S}(M, N) \rightarrow \operatorname{Tor}_{1}^{R}(M, N) \rightarrow 0 .
\end{aligned}
$$

\section{Vanishing of Ext and Tor over Arbitrary Local Gorenstein Rings}

In this section we prove what type of duality between the vanishing of Ext and Tor holds over arbitrary local Gorenstein rings. It is possible an even stronger result is true, as we discuss in Section 4, but the main result of this section is what is true 'on the surface'. In particular, Theorem 2.1 states that one can flip the arguments in vanishing Ext modules 'up to duals'.

Theorem 2.1. Let $R$ be a local Gorenstein ring, and let $M$ and $N$ be finitely generated maximal Cohen-Macaulay $R$-modules. Then the following are equivalent:

(1) $\operatorname{Tor}_{i}^{R}(M, N)=0$ for all $i \gg 0$,

(2) $\operatorname{Ext}_{R}^{i}\left(M, N^{*}\right)=0$ for all $i \gg 0$, and

(3) $\operatorname{Ext}_{R}^{i}\left(N, M^{*}\right)=0$ for all $i \gg 0$.

Proof. Suppose we have shown that (1) and (2) are equivalent. By replacing (1) by the equivalent condition that $\operatorname{Tor}_{i}^{R}(N, M)=0$ for all $i \gg 0$, we see then that (1) is equivalent to (3). Hence it suffices to prove (1) and (2) are equivalent, and for this we only need to assume that $N$ is maximal Cohen-Macaulay.

We induce upon the dimension of $R$, say $d$. If $d=0$, then $\operatorname{Ext}_{R}^{i}\left(M, N^{*}\right)$ is the Matlis dual of $\operatorname{Tor}_{i}^{R}(M, N)$, so the result is immediate in this case. 
Now assume that $d>0$. Choose $x \in R$ a non-zerodivisor on $M_{d}, N, N^{*}$, and $R$. We have

$$
\begin{array}{ll}
\operatorname{Tor}_{i}^{R}(M, N)=0 & \text { for all } i \gg 0 ; \\
\operatorname{Tor}_{i}^{R}\left(M_{d}, N\right)=0 & \text { for all } i \gg 0 ;
\end{array}
$$

and from the long exact sequence of Tor coming from the short exact sequence $0 \rightarrow N \stackrel{x}{\rightarrow} N \rightarrow N / x N \rightarrow 0$ and Nakayama's lemma,

$$
\Longleftrightarrow \operatorname{Tor}_{i}^{R}\left(M_{d}, N / x N\right)=0 \quad \text { for all } i \gg 0 \text {; }
$$

and by the standard isomorphisms $\operatorname{Tor}_{i}^{R}\left(M_{d}, N / x N\right) \simeq \operatorname{Tor}_{i}^{R /(x)}\left(M_{d} / x M_{d}\right.$, $N / x N)$,

$$
\Longleftrightarrow \operatorname{Tor}_{i}^{R /(x)}\left(M_{d} / x M_{d}, N / x N\right)=0 \quad \text { for all } i \gg 0 ;
$$

by the induction hypothesis,

$$
\Longleftrightarrow \operatorname{Ext}_{R /(x)}^{i}\left(M_{d} / x M_{d},(N / x N)^{*}\right)=0 \quad \text { for all } i \gg 0 ;
$$

and now since $N$ is maximal Cohen-Macaulay and $R$ is Gorenstein, $N^{*} / x N^{*} \simeq$ $(N / x N)^{*}$ (where the second module is $\operatorname{Hom}_{R /(x)}(N / x N, R /(x))$ ), thus,

$$
\Longleftrightarrow \operatorname{Ext}_{R /(x)}^{i}\left(M_{d} / x M_{d}, N^{*} / x N^{*}\right)=0 \quad \text { for all } i \gg 0 ;
$$

by the isomorphisms [5] $\operatorname{Ext}_{R}^{i}\left(M_{d}, N^{*} / x N^{*}\right) \simeq \operatorname{Ext}_{R /(x)}^{i}\left(M_{d} / x M_{d}, N^{*} / x N^{*}\right)$,

$$
\Longleftrightarrow \operatorname{Ext}_{R}^{i}\left(M_{d}, N^{*} / x N^{*}\right)=0 \quad \text { for all } i \gg 0 ;
$$

and now from the long exact sequence of Ext coming from the short exact sequence $0 \rightarrow N^{*} \stackrel{x}{\rightarrow} N^{*} \rightarrow N^{*} / x N^{*} \rightarrow 0$ and Nakayama's lemma,

$$
\begin{array}{ll}
\Longleftrightarrow \operatorname{Ext}_{R}^{i}\left(M_{d}, N^{*}\right)=0 & \text { for all } i \gg 0 ; \\
\operatorname{Ext}_{R}^{i}\left(M, N^{*}\right)=0 & \text { for all } i \gg 0 .
\end{array}
$$

Remark 2.2. Suppose $M$ and $N$ are maximal Cohen-Macaulay modules over the local Gorenstein ring $R$. Then

$$
\operatorname{Ext}_{R}^{i}(M, N) \simeq \operatorname{Ext}_{R}^{i}\left(N^{*}, M^{*}\right) .
$$

This isomorphism can be seen as follows: suppose that $i=1$. As $M$ and $N$ are maximal Cohen-Macaulay, they are reflexive, so short exact sequences $0 \rightarrow N \rightarrow T \rightarrow M \rightarrow 0$ dualize to short exact sequences $0 \rightarrow M^{*} \rightarrow$ $T^{*} \rightarrow N^{*} \rightarrow 0$ and vice-versa. The Yoneda definition of $\mathrm{Ext}^{1}$ then gives the isomorphism. 
For $i>1$ we have

$$
\begin{array}{rlrl}
\operatorname{Ext}_{R}^{i}(M, N) & \simeq \operatorname{Ext}_{R}^{1}\left(M_{i-1}, N\right) & \\
& \simeq \operatorname{Ext}_{R}^{1}\left(N^{*}, M_{i-1}^{*}\right) & & \text { by the } i=1 \text { case } \\
& \simeq \operatorname{Ext}_{R}^{1}\left(N^{*},\left(M^{*}\right)_{-i+1}\right) & & \text { by (2) of Lemma } 1.1 \\
& \simeq \operatorname{Ext}_{R}^{i}\left(N^{*}, M^{*}\right) & & \text { by (1.2). }
\end{array}
$$

Presumably, one can directly prove this remark using the Yoneda definition of Ext and the fact that both $M$ and $N$ are maximal Cohen-Macaulay.

Below is an example showing that the hypothesis that $N$ is maximal CohenMacaulay in the equivalence of (1) and (2) in Theorem 2.1 cannot be dropped.

Example 2.3. Let $R$ be the 3-dimensional hypersurface $k[[W, X, Y, Z]] /$ $(W X-Y Z)$, and set $M:=k$ and $N:=\operatorname{coker}\left(\begin{array}{l}w \\ x \\ y \\ z\end{array}\right)$. Then $\operatorname{pd}_{R} N=1$ (but $\left.\operatorname{pd}_{R} N^{*}=\infty\right)$, so we have $\operatorname{Tor}_{i}^{R}(M, N)=0$ for all $i \gg 0$. However, $\operatorname{Ext}_{R}^{4}\left(M, N^{*}\right) \neq 0$. By what is shown in the next section, $R$ is an AB ring. If it were the case that $\operatorname{Ext}_{R}^{i}\left(M, N^{*}\right)$ is zero for all $i \gg 0$ then, as $R$ is $\mathrm{AB}$, Proposition 3.2 shows then that $\operatorname{Ext}_{R}^{i}\left(M, N^{*}\right)=0$ for all $i>\operatorname{dim} R$, which would be a contradiction.

\section{AB Rings}

Let $R$ be a commutative ring. We define the Ext-index of $R$ to be

$$
\sup \left\{n \mid \operatorname{Ext}_{R}^{i}(M, N)=0 \text { for all } i>n \text { and } \operatorname{Ext}_{R}^{n}(M, N) \neq 0\right\},
$$

where the sup is taken over all pairs of finitely generated $R$-modules $(M, N)$ with $\operatorname{Ext}_{R}^{i}(M, N)=0$ for all $i \gg 0$.

Definition 3.1. If $R$ is a local Gorenstein ring of finite Ext-index, we say that $R$ is an $A B$ ring.

We will prove that all complete intersections are $\mathrm{AB}$ rings. More generally, it is obvious that $R$ is an $\mathrm{AB}$ ring if $\hat{R}$ is (where $\hat{R}$ is the completion of $R$ ), and we show (3.3) that if $R$ is an $\mathrm{AB}$ ring and $x_{1}, \ldots, x_{c}$ is a regular sequence, then $R /\left(x_{1}, \ldots, x_{c}\right)$ is also an $\mathrm{AB}$ ring. The class of $\mathrm{AB}$ rings also includes local Gorenstein rings of 'minimal' multiplicity $\operatorname{embdim}(R)-\operatorname{dim}(R)+2$ (see 3.6).

Proposition 3.2. Suppose that $R$ is an $A B$ ring. Then the Ext-index of $R$ equals $\operatorname{dim} R$. 
Proof. Let $n$ denote the Ext-index of $R$ and $d$ the dimension of $R$. Let $x_{1}, \ldots, x_{d}$ be a maximal $R$-sequence. Set $M:=R /\left(x_{1}, \ldots, x_{d}\right)$ and $N=k$, the residue field of $R$. Then $\operatorname{Ext}_{R}^{i}(M, N)=0$ for $i>d$ and $\operatorname{Ext}_{R}^{d}(M, N) \simeq$ $k \neq 0$. Hence $n \geq d$.

Suppose that $n>d$. There exists a pair of finitely generated $R$-modules $(M, N)$ such that $\operatorname{Ext}_{R}^{i}(M, N)=0$ for $i>n$ and $\operatorname{Ext}_{R}^{n}(M, N) \neq 0$. We have the isomorphisms $\operatorname{Ext}_{R}^{i+1}\left(\left(M_{d}\right)_{-d-1}, N\right) \simeq \operatorname{Ext}_{R}^{i-d}\left(M_{d}, N\right) \simeq$ $\operatorname{Ext}_{R}^{i}(M, N)$ for $i>d$. Hence $\operatorname{Ext}^{i}\left(\left(M_{d}\right)_{-d-1}, N\right)=0$ for $i>n+1$ and $\operatorname{Ext}^{n+1}\left(\left(M_{d}\right)_{-d-1}, N\right) \neq 0$, which contradicts the definition of $n$. Therefore $n=d$.

We of course have the dual notion of Tor-index. If $R$ is local Gorenstein with finite Tor-index, then it is also equal to $\operatorname{dim} R$, by an argument analogous to that of 3.2.

Another related property of rings we are interested in is the following. We say that $\operatorname{Ext}_{R}^{*}(M, N)$ has a gap of length $t$ if for some $n \geq 0, \operatorname{Ext}_{R}^{i}(M, N)=0$ for $n+1 \leq i \leq n+t$, $\operatorname{but}_{\operatorname{Ext}_{R}^{n}}(M, N)$ and $\operatorname{Ext}_{R}^{n+t+1}(M, N)$ are both nonzero. We have the analogous notion of gap for $\operatorname{Tor}_{*}^{R}(M, N)$. (We allow gaps of length 0.) We set

$$
\operatorname{Ext-gap}(R):=\sup \left\{\begin{array}{l|l}
t \in \mathrm{N} & \begin{array}{l}
\operatorname{Ext}_{R}^{*}(M, N) \text { has a gap of length } t \\
\text { for finite } R \text {-modules } M \text { and } N
\end{array}
\end{array}\right\},
$$

and

$$
\operatorname{Tor-gap}(R):=\sup \left\{\begin{array}{l|l}
t \in \mathrm{N} & \begin{array}{l}
\operatorname{Tor}_{*}^{R}(M, N) \text { has a gap of length } t \\
\text { for finite } R \text {-modules } M \text { and } N
\end{array}
\end{array}\right\} .
$$

We say that $R$ is Ext-bounded if it has finite Ext-gap. Similarly, we say $R$ is Tor-bounded if it has finite Tor-gap.

We list some elementary properties involving finite Ext-index, Tor-index, Ext-boundedness and Tor-boundedness for local Gorenstein rings.

Proposition 3.3. Let $x$ be a non-zerodivisor of the d-dimensional local Gorenstein ring $R$. Then

(1) $R$ is an $A B$ ring if and only if $R /(x)$ is an $A B$ ring.

(2) $R$ has finite Tor-index if and only if $R /(x)$ does.

(3) $R$ is Ext-bounded if and only if $R /(x)$ is.

(4) $R$ is Tor-bounded if and only if $R /(x)$ is.

Proof. (1). Suppose that $R$ is an $\mathrm{AB}$ ring. Let $M$ and $N$ be finitely generated $R /(x)$-modules such that $\operatorname{Ext}_{R /(x)}^{i}(M, N)=0$ for all $i \gg 0$. By the change 
of rings long exact sequence of Ext (1.3) we conclude that $\operatorname{Ext}_{R}^{i}(M, N)=0$ for all $i \gg 0$, and so $\operatorname{Ext}_{R}^{i}(M, N)=0$ for all $i>d$. Looking at (1.3) again, we see that $\operatorname{Ext}_{R /(x)}^{i}(M, N) \simeq \operatorname{Ext}_{R /(x)}^{i+2}(M, N)$ for $i>d-1$. But as $\operatorname{Ext}_{R /(x)}^{i}(M, N)=0$ for all $i \gg 0$, we have $\operatorname{Ext}_{R /(x)}^{i}(M, N)=0$ for all $i>d-1$. Hence $R /(x)$ is an $\mathrm{AB}$ ring.

Now suppose that $R /(x)$ is an $\mathrm{AB}$ ring, and let $M$ and $N$ be finitely generated $R$-modules such that $\operatorname{Ext}_{R}^{i}(M, N)=0$ for all $i \gg 0$. We have the isomorphisms

$$
\operatorname{Ext}_{R}^{i}(M, N) \simeq \operatorname{Ext}_{R}^{i-d}\left(M_{d}, N\right) \simeq \operatorname{Ext}_{R}^{i}\left(M_{d}, N_{d}\right)
$$

which are valid for $i>d$, the second one being that of (1.2). The short exact sequence $0 \rightarrow N_{d} \stackrel{x}{\rightarrow} N_{d} \rightarrow N_{d} / x N_{d} \rightarrow 0$ gives rise to the the long exact sequence of Ext

$$
\cdots \rightarrow \operatorname{Ext}_{R}^{i}\left(M_{d}, N_{d}\right) \stackrel{x}{\rightarrow} \operatorname{Ext}_{R}^{i}\left(M_{d}, N_{d}\right) \rightarrow \operatorname{Ext}_{R}^{i}\left(M_{d}, N_{d} / x N_{d}\right) \rightarrow \cdots .
$$

Since $\operatorname{Ext}_{R}^{i}\left(M_{d}, N_{d}\right)=0$ for all $i \gg 0$, we see that $\operatorname{Ext}_{R}^{i}\left(M_{d}, N_{d} / x N_{d}\right)=0$ for all $i \gg 0$. We have the isomorphisms [5]

$$
\operatorname{Ext}_{R /(x)}^{i}\left(M_{d} / x M_{d}, N_{d} / x N_{d}\right) \simeq \operatorname{Ext}_{R}^{i}\left(M_{d}, N_{d} / x N_{d}\right)
$$

for all $i \geq 0$. Hence $\operatorname{Ext}_{R /(x)}^{i}\left(M_{d} / x M_{d}, N_{d} / x N_{d}\right)=0$ for all $i \gg 0$, which means that $\operatorname{Ext}_{R /(x)}^{i}\left(M_{d} / x M_{d}, N_{d} / x N_{d}\right)=0$ for all $i>d-1$, since $R /(x)$ is an $\mathrm{AB}$ ring. Therefore $\operatorname{Ext}_{R}^{i}\left(M_{d}, N_{d} / x N_{d}\right)=0$ for all $i>d-1$. By (3.3.1) and Nakayama's Lemma, we conclude that $\operatorname{Ext}_{R}^{i}\left(M_{d}, N_{d}\right)=0$ for all $i>d-1$, and so $\operatorname{Ext}_{R}^{i}(M, N)=0$ for all $i>d$. Therefore $R$ is an AB ring.

The proof of (2) is exactly analogous to the proof of (1), using (1.4) and a long exact sequence of Tor this time.

(3). Assume that $e:=\operatorname{Ext-gap}(R)<\infty$. Let $M$ and $N$ be finitely generated $R /(x)$-modules such that $\operatorname{Ext}_{R /(x)}^{i}(M, N)=0$ for $n \leq i \leq n+e+1$, some $n \geq 1$. The change of rings long exact sequence of Ext (1.3) shows that $\operatorname{Ext}_{R}^{i}(M, N)=0$ for $n+1 \leq i \leq n+e+1$. Since $\operatorname{Ext-gap}(R)=e$ we have $\operatorname{Ext}_{R}^{i}(M, N)=0$ for all $i>n$. Another look at (1.3) shows that $\operatorname{Ext}_{R /(x)}^{i}(M, N) \simeq \operatorname{Ext}_{R /(x)}^{i+2}(M, N)$ for all $i>n-1$. Since $\operatorname{Ext}_{R /(x)}^{i}(M, N)=$ 0 for $i=n, n+1$, we see then that $\operatorname{Ext}_{R /(x)}^{i}(M, N)=0$ for $i>n-1$ Hence $\operatorname{Ext}-\operatorname{gap}(R /(x)) \leq e+1$.

Now assume that $e:=\operatorname{Ext}-\operatorname{gap}(R /(x))<\infty$. Suppose that $M$ and $N$ are finitely generated $R$-modules with $\operatorname{Ext}_{R}^{i}(M, N)=0$ for $n \leq i \leq n+d+e+1$, some $n \geq 1$. We have $\operatorname{Ext}_{R}^{i}\left(M_{d}, N_{d}\right) \simeq \operatorname{Ext}_{R}^{i}(M, N)=0$ for $n+d \leq i \leq n+$ $d+e+1$. Therefore, from (3.3.1), we get $\operatorname{Ext}_{R}^{i}\left(M_{d}, N_{d} / x N_{d}\right)=0$ for $n+d \leq$ 
$i \leq n+d+e$. Equivalently, $\operatorname{Ext}_{R /(x)}^{i}\left(M_{d} / x M_{d}, N_{d} / x N_{d}\right)=0$ for $n+d \leq i \leq$ $n+d+e$. Since $\operatorname{Ext}-\operatorname{gap}(R /(x))=e, \operatorname{Ext}_{R /(x)}^{i}\left(M_{d} / x M_{d}, N_{d} / x N_{d}\right)=0$ for all $i \geq n+d$, which implies, by (3.3.1) and Nakayama's lemma, $\operatorname{Ext}_{R}^{i}\left(M_{d}, N_{d}\right)=$ 0 for all $i \geq n+d$, which means $\operatorname{Ext}_{R}^{i}(M, N)=0$ for all $i \geq n$. Therefore $\operatorname{Ext}-\operatorname{gap}(R) \leq d+e+1$.

The proof of (4) is similar to the proof of (3).

Theorem 3.4. Assume that $R$ is a local Gorenstein ring. Then

(1) $R$ is an $A B$ ring if and only if it has finite Tor-index;

(2) $R$ is Ext-bounded if and only if it is Tor-bounded;

(3) if $R$ is Ext-bounded, then it is an $A B$ ring.

Proof. We first prove (1). Choose a maximal regular sequence in $R$ and let $I$ be the ideal generated by this sequence. Proposition 3.3 states that $R$ is an $\mathrm{AB}$ ring if and only if $R / I$ is an $\mathrm{AB}$ ring, and $R$ has finite Tor-index if and only if $R / I$ has finite Tor-index. Hence it suffices to prove (1) in case $R$ is 0-dimensional. In this case $\operatorname{Ext}_{R}^{i}(M, N)$ is the Matlis dual of $\operatorname{Tor}_{i}^{R}\left(M, N^{*}\right)$ so that the vanishing of one implies the vanishing of the other. This proves (1).

Statement (2) is proved in a similar manner, using Proposition 3.3.

We prove (3). Assume $R$ is Ext-bounded. We prove that $R$ has finite Torindex. Let $d$ denote the dimension of $R$ and $e:=\operatorname{Ext}-g a p(R)$, and suppose that for finite $R$-modules $M$ and $N \operatorname{Tor}_{i}^{R}(M, N)=0$ for all $i \gg 0$. Let $b=$ $d$ - depth $M$ so that $M_{b}$ is maximal Cohen-Macaulay. Choose $n$ largest such that $\operatorname{Tor}_{n}^{R}\left(M_{b}, N\right) \neq 0$. Using $t=e+n+3$ in (5)(i) of Lemma 1.1, we have $\operatorname{Ext}_{R}^{i}\left(\left(M_{b}^{*}\right)_{-e-n-3}, N\right) \simeq \operatorname{Tor}_{e+n+2-i}^{R}\left(M_{b}, N\right)=0$ for $1 \leq i \leq e+1$. Hence we have a gap of zero Ext larger than $e$. Therefore $\operatorname{Ext}_{R}^{i}\left(\left(M_{b}^{*}\right)_{-e-n-3}, N\right)=0$ for all $i \geq 1$, which forces $n=0$. Thus $\operatorname{Tor}_{i}^{R}(M, N)=0$ for all $i>d$.

The following Corollary is an almost immediate consequence of Proposition 3.4, as regular local rings are clearly Ext-bounded.

Corollary 3.5. Let $R$ be a local Gorenstein ring. If $R$ is a complete intersection, then $R$ is Ext-bounded. In particular, $R$ is an $A B$ ring.

Proof. Since $R \hookrightarrow \hat{R}$ is a faithfully flat extension, $\operatorname{Ext}_{R}^{i}(M, N)=0$ if and only if $\operatorname{Ext}_{\hat{R}}^{i}(\hat{M}, \hat{N})=0$ and so $R$ is an $\mathrm{AB}$ ring if $\hat{R}$ is. Therefore we may without loss of generality assume that $R=S /\left(x_{1}, \ldots, x_{c}\right)$ where $S$ is a regular local ring and $x_{1}, \ldots, x_{c}$ is an $S$-regular sequence. By Proposition 3.3 it suffices to prove that $S$ is Ext-bounded. But this is trivial as every finitely generated module over $S$ has projective dimension $\leq \operatorname{dim} S$, so that $\operatorname{Ext}_{R}^{i}(M, N)=0$ for $i>\operatorname{dim} S$ and Ext-gaps can occur of length no longer than $\operatorname{dim} S-2$. 
It could be that all local Gorenstein rings are $\mathrm{AB}$ rings; we have no counterexample. The class of $\mathrm{AB}$ rings is strictly bigger than the class of complete intersections as the next theorem proves, albeit for rather strong reasons. (See, however, Theorem 3.8 and Propostion 4.4.)

THEOREM 3.6. Let $(R, \mathfrak{m}, k)$ be a local Gorenstein ring with multiplicity equal to embdim $R-\operatorname{dim} R+2$. Assume that $\operatorname{embdim} R>\operatorname{dim} R+2$ (so that $R$ is not a complete intersection). Then for finitely generated $R$-modules $M$ and $N, \operatorname{Ext}_{R}^{i}(M, N)=0$ for all $i \gg 0$ if and only if either $M$ or $N$ has finite projective dimension. In particular, $R$ is an $A B$ ring.

Proof. We induce on $d:=\operatorname{dim} R$.

$d=0$. In this case by duality we have that $\operatorname{Tor}_{i}^{R}\left(M, N^{*}\right)=0$ for all $i \gg 0$. Replace $M$ by a high enough syzygy such that $\operatorname{Tor}_{3}^{R}\left(M, N^{*}\right)=$ $\operatorname{Tor}_{4}^{R}\left(M, N^{*}\right)=\operatorname{Tor}_{5}^{R}\left(M, N^{*}\right)=0$. Now the following lemma, 3.7, says that either $M$ or $N$ is free.

$d>0$. By replacing $M$ and $N$ by syzygies we can assume they are both maximal Cohen-Macaulay. Choose a minimal generator $x$ of the maximal ideal of $R$ such that the multiplicity of $R /(x)$ is embdim $R /(x)-\operatorname{dim} R /(x)+2$, and $x$ is a non-zerodivisor on both $M$ and $N$. Once again we use the fact that $\operatorname{Ext}_{R}^{i}(M, N)=0$ for all $i \gg 0$ if and only if $\operatorname{Ext}_{R /(x)}^{i}(M / x M, N / x N)=0$ for all $i \gg 0$. By induction either $M / x M$ or $N / x N$ has finite projective dimension over $R /(x)$. But then either $M$ or $N$ has finite projective dimension over $R$.

LeMma 3.7. Let $(R, \mathfrak{m}, k)$ be a 0 -dimensional local Gorenstein ring with multiplicity embdim $R+2$. Assume embdim $R>2$ (so that $R$ is not a complete intersection). Let $M$ and $N$ be finitely generated $R$-modules. Then $\operatorname{Tor}_{R}^{3}(M, N)=\operatorname{Tor}_{R}^{4}(M, N)=\operatorname{Tor}_{R}^{5}(M, N)=0$ if and only if either $M$ or $N$ is free.

Proof. Let $n$ denote the embedding dimension of $R$. Assume that $M$ is not free. If $k$ is a summand of $M_{i}$ for any $0 \leq i \leq 4$, then we get right away that $N$ is free, since $\operatorname{Tor}_{1}^{R}\left(M_{i}, N\right)=\operatorname{Tor}_{i+1}^{R}(M, N)=0$ would then imply $\operatorname{Tor}_{1}^{R}(k, N)=0$. Therefore assume $k$ is not a summand of $M_{i}$ for all $0 \leq i \leq 4$. Replace $M$ by its first syzygy. Then as $M \subseteq \mathfrak{m} F$, for $F$ a free module, we have $\mathfrak{m}^{2} M=0$ (since $\left.\mathfrak{m}^{3}=0\right)$. Let $b_{i}$ denote the $i$ th Betti number of $M$ and $s:=\operatorname{dim}_{k} \mathfrak{m} M$. Then, as in Lescot's paper [3, Lemma 3.3], $M$ is 3-exceptional and $b_{1}=n b_{0}-s, b_{2}=b_{0}\left(n^{2}-1\right)-s n$ and $b_{3}=b_{0}\left(n^{3}-2 n\right)-s\left(n^{2}-1\right)$.

Now suppose that $N$ is also not free. Also replace $N$ by its first syzygy, so that $\mathfrak{m}^{2} N=0$. Write $\mathfrak{m} N \simeq k^{d}$. We have a short exact sequence $0 \rightarrow k^{d} \rightarrow$ $N \rightarrow k^{c} \rightarrow 0$, where $c$ is the minimal number of generators of $N$. Applying $M \otimes_{R}$ to this short exact sequence and using the fact that $\operatorname{Tor}_{R}^{1}(M, N)=$ $\operatorname{Tor}_{R}^{2}(M, N)=\operatorname{Tor}_{R}^{3}(M, N)=0$ we get $c b_{2}=d b_{1}$ and $c b_{3}=d b_{2}$. Letting 
$\alpha:=d / c$ we can write these equations as $b_{2}=\alpha b_{1}$ and $b_{3}=\alpha b_{2}=\alpha^{2} b_{1}$. Substituting for the $b_{i}$ we get

$$
\begin{aligned}
b_{0}\left(n^{2}-1\right)-s n & =\alpha\left(n b_{0}-s\right) \\
b_{0}\left(n^{3}-2 n\right)-s\left(n^{2}-1\right) & =\alpha^{2}\left(n b_{0}-s\right) .
\end{aligned}
$$

After rearranging we arrive at

$$
\begin{aligned}
b_{0}\left(n^{2}-\alpha n-1\right) & =s(n-\alpha) \\
b_{0}\left(n^{3}-\alpha^{2} n-2 n\right) & =s\left(n^{2}-\alpha^{2}-1\right) .
\end{aligned}
$$

Now cross multiplying, cancelling off the $b_{0} s$ terms, and simplifying we are left with the condition $\alpha^{2}-n \alpha+1=0$. This says that $\alpha \in \mathrm{Q}$ is algebraic over Z. Hence $\alpha$ is an integer, and the only choice is $\alpha=1$. But then $n=2$, which is a contradiction. Hence $N$ must be free.

There also exist $\mathrm{AB}$ rings which are not complete intersections and which have multiplicity $>\operatorname{embdim} R-\operatorname{dim} R+2$ :

THEOREm 3.8. Suppose that $\left(R, \mathfrak{m}_{R}, k\right)$ and $\left(S, \mathfrak{m}_{S}, k\right)$ are local rings essentially of finite type over the same field $k$. Set $A:=\left(R \otimes_{k} S\right)_{P}$, where $P:=\mathfrak{m}_{R} \otimes_{k} S+R \otimes_{k} \mathfrak{m}_{S}$. If both $R$ and $S$ are Gorenstein then $A$ is Gorenstein, and the multiplicity of $A$ is $>\operatorname{embdim} A-\operatorname{dim} A+2$. If $R$ is an $A B$ ring and $S$ is the quotient of a regular local ring by a regular sequence (so that $S$ is a complete intersection), then $A$ is an $A B$ ring; $A$ is not a complete intersection if $R$ is not.

Proof. Suppose that $R$ and $S$ are Gorenstein. Obviously $A$ is Noetherian, being a localization of a finitely generated $k$-algebra. By applying [7] we have, moreover, that it is Gorenstein. Proposition 4.3 of the next section shows that there exist finitely generated $A$-modules $M$ and $N$ both of infinite projective dimension over $A$ such that $\operatorname{Ext}_{A}^{i}(M, N)=0$ for all $i>\operatorname{dim} A$. It follows from Theorem 3.6 that the multiplicity of $A$ is larger than embdim $A-\operatorname{dim} A+2$.

Suppose now that $R$ is an $\mathrm{AB}$ ring and that $S$ is the quotient of the regular local ring $\left(T, \mathfrak{m}_{T}, k\right)$ by the $T$-regular sequence $\mathbf{x}:=x_{1}, \ldots, x_{c}$. Clearly $A$ is isomorphic to $\left(R \otimes_{k} T\right)_{P^{\prime}} /(1 \otimes \mathbf{x})$ where $P^{\prime}:=\mathfrak{m}_{R} \otimes_{k} T+R \otimes_{k} \mathfrak{m}_{T}$ and $1 \otimes \mathbf{x}$ is the regular sequence $1 \otimes x_{1}, \ldots, 1 \otimes x_{c}$. Hence by Proposition 3.3, $A$ is an $\mathrm{AB}$ ring if and only if $\left(R \otimes_{k} T\right)_{P^{\prime}}$ is an $\mathrm{AB}$ ring. But now going modulo $1 \otimes y_{1}, \ldots, 1 \otimes y_{d}$ where $d:=\operatorname{dim} T$ and $y_{1}, \ldots, y_{d}$ a regular system of parameters of $T$ shows, again by Proposition 3.3, that $\left(R \otimes_{k} T\right)_{P^{\prime}}$ is an AB ring if and only if $R$ is an $\mathrm{AB}$ ring.

Recall that a local ring $(A, \mathfrak{m}, k)$ is a complete intersection precisely when

$$
\operatorname{dim}_{k} H_{1}(A)=\operatorname{embdim} A-\operatorname{dim} A,
$$


where $H_{1}(A)$ is the first Koszul homology module on a system of generators for the maximal ideal of $A$. In the current situation we have $\operatorname{dim}_{k} H_{1}(A)=$ $\operatorname{dim}_{k} H_{1}(R)+\operatorname{dim}_{k} H_{1}(S), \operatorname{embdim} A=\operatorname{embdim} R+\operatorname{embdim} S$, and $\operatorname{dim} A=$ $\operatorname{dim} R+\operatorname{dim} S$. Hence if $R$ is not a complete intersection, then $\operatorname{dim}_{k} H_{1}(R)>$ embdim $R-\operatorname{dim} R$, so that $\operatorname{dim}_{k} H_{1}(A)>\operatorname{embdim} A-\operatorname{dim} A$.

\section{Vanishing of Ext and Tor over AB Rings}

In this section we prove that $\mathrm{AB}$ rings are a class which gives the duality of vanishing Ext discussed in the introduction. Our main theorem states:

Theorem 4.1. Suppose that $R$ is an $A B$ ring, and let $M$ and $N$ be finitely generated $R$-modules. Then

$$
\operatorname{Ext}_{R}^{i}(M, N)=0 \quad \text { for all } i \gg 0
$$

if and only if

$$
\operatorname{Ext}_{R}^{i}(N, M)=0 \quad \text { for all } i \gg 0
$$

Proof. First assume the theorem is true if both $M$ and $N$ are maximal Cohen-Macaulay. For the general case, take syzygies $M_{m}$ and $N_{n}(m, n \geq 0)$ of $M$ and $N$, respectively, which are maximal Cohen-Macaulay. We have $\operatorname{Ext}_{R}^{i}(M, N)=0$ for all $i \gg 0$ if and only if $\operatorname{Ext}_{R}^{i}\left(M_{m}, N\right)=0$ for all $i \gg 0$ and by (1.2) this is equivalent to $\operatorname{Ext}_{R}^{i}\left(M_{m}, N_{n}\right)=0$ for all $i \gg 0$. Thus $\operatorname{Ext}_{R}^{i}(M, N)=0$ for all $i \gg 0$ if and only if $\operatorname{Ext}_{R}^{i}\left(M_{m}, N_{n}\right)=0$ for all $i \gg 0$, and so the theorem holds generally.

Now suppose that $M$ and $N$ are maximal Cohen-Macaulay and $\operatorname{Ext}_{R}^{i}(M, N)$ $=0$ for all $i \gg 0$. Then for all $t \geq 1, \operatorname{Ext}_{R}^{i}\left(M_{-t}, N\right)=0$ for all $i \gg 0$. Since $R$ is an $\mathrm{AB}$ ring, it follows from Proposition 3.2 that for all $t \geq 1$ and all $i>d:=$ $\operatorname{dim}(R), \operatorname{Ext}_{R}^{i}\left(M_{-t}, N\right)=0$. However, $\operatorname{Ext}_{R}^{i}\left(M_{-t}, N\right) \simeq \operatorname{Ext}_{R}^{1}\left(M_{i-t-1}, N\right)$. Hence for all $t \geq 1$ and all $i>d$, $\operatorname{Ext}_{R}^{1}\left(M_{i-t+1}, N\right)=0$. By varying $i$ and $t$, we obtain that $\operatorname{Ext}_{R}^{1}\left(M_{-t}, N\right)=0$ for all $t \geq 1$. Therefore by (5)(i) of Lemma 1.1, $\operatorname{Tor}_{t-2}^{R}\left(M^{*}, N\right)=\operatorname{Tor}_{t-2}^{R}\left(N, M^{*}\right)=0$ for all $t \geq 3$. Applying Theorem 2.1 then shows that $\operatorname{Ext}_{R}^{i}(N, M)=0$ for all $i \gg 0$.

As an immediate corollary, we have an analogue of Theorem 2.1

Corollary 4.2. Suppose that $R$ is an $A B$ ring, and let $M$ and $N$ be finitely generated maximal Cohen-Macaulay $R$-modules. Then the following are equivalent:

(1) $\operatorname{Tor}_{i}^{R}(M, N)=0$ for all $i \gg 0$,

(2) $\operatorname{Ext}_{R}^{i}\left(M^{*}, N\right)=0$ for all $i \gg 0$, and 
(3) $\operatorname{Ext}_{R}^{i}\left(N^{*}, M\right)=0$ for all $i \gg 0$.

Proof. Due to the natural symmetry in Tor, it suffices to prove just the equivalence between (1) and (2), and for this we only need to assume that $M$ is maximal Cohen-Macaulay.

We have

$$
\begin{aligned}
\operatorname{Tor}_{i}^{R}(M, N)=0 \text { for all } i \gg 0 & \Longleftrightarrow \operatorname{Tor}_{i}^{R}(N, M)=0 \text { for all } i \gg 0 \\
& \Longleftrightarrow \operatorname{Ext}_{R}^{i}\left(N, M^{*}\right)=0 \text { for all } i \gg 0 \quad \text { by } 2.1 \\
& \Longleftrightarrow \operatorname{Ext}_{R}^{i}\left(M^{*}, N\right)=0 \text { for all } i \gg 0 \text { by } 4.1
\end{aligned}
$$

If we strengthen the condition on the symmetry in the vanishing of Ext we get an equivalent definition of $\mathrm{AB}$ ring.

Proposition 4.3. Let $(R, \mathfrak{m}, k)$ be a local ring of dimension $d$. Then the following are equivalent:

(1) $R$ is an $A B$ ring;

(2) For finitely generated $R$-modules $M$ and $N$, $\operatorname{Ext}_{R}^{i}(M, N)=0$ for $i>d$ if and only if $\operatorname{Ext}_{R}^{i}(N, M)=0$ for $i>d$.

Proof. $(1) \Longrightarrow(2)$. This is simply Theorem 4.1 coupled with Proposition 3.2 .

For (2) $\Longrightarrow(1)$, we get right away that $R$ is Gorenstein from the hypothesis with $M:=R$ and $N:=k$.

For the remainder of the proof we will use the following fact. If $M$ and $N$ are finitely generated $R$-modules, then $\operatorname{Ext}_{R}^{i}(M, N) \simeq \operatorname{Ext}_{R}^{i+n}\left(M, N_{n}\right)$ for $i>d$ and $n \geq 0$. This follows from the isomorphisms $\operatorname{Ext}_{R}^{i}(M, N) \simeq$ $\operatorname{Ext}_{R}^{i-d}\left(M_{d}, N\right) \simeq \operatorname{Ext}^{i-d+n}\left(M_{d}, N_{n}\right) \simeq \operatorname{Ext}_{R}^{i+n}\left(M, N_{n}\right)$ for $i>d$ and $n \geq 0$, where the middle isomorphism is formula (1.2) of Section 1.

Suppose that $\operatorname{Ext}_{R}^{i}(M, N)=0$ for all $i>d+n$, some fixed $n \geq 1$. Then we have

$$
\begin{array}{lll} 
& \operatorname{Ext}_{R}^{i}\left(M_{n}, N\right)=0 & \text { for } i>d ; \\
\operatorname{Ext}_{R}^{i}\left(N, M_{n}\right)=0 & \text { for } i>d & \text { by hypothesis; } \\
\Longrightarrow & \operatorname{Ext}_{R}^{i+n}\left(N, M_{n}\right)=0 & \text { for } i>d ; \\
\operatorname{Ext}_{R}^{i}(N, M)=0 & \text { for } i>d \quad \text { by the isomorphism above; } \\
\operatorname{Ext}_{R}^{i}(M, N)=0 & \text { for } i>d \quad \text { by hypothesis. }
\end{array}
$$


Our final proposition in this section is an observation that there are circumstances other than where one module has finite projective dimension or where the ring is a complete intersection in which all large Ext modules vanish.

Proposition-Example 4.4. Let $\left(R, \mathfrak{m}_{R}, k\right)$ and $\left(S, \mathfrak{m}_{S}, k\right)$ be two local Gorenstein rings essentially of finite type over the same field $k$, and let $M_{R}$ be a finitely generated $R$-module and $N_{S}$ a finitely generated $S$-module. Set $A:=\left(R \otimes_{k} S\right)_{P}$ where $P:=\left(\mathfrak{m}_{R} \otimes_{k} S+R \otimes_{k} \mathfrak{m}_{S}\right), M:=\left(M_{R} \otimes_{k} S\right)_{P}$ and $N:=\left(R \otimes_{k} N_{S}\right)_{P}$. Then $A$ is a local Gorenstein ring, $M$ has finite projective dimension over $A$ if and only if $M_{R}$ has finite projective dimension over $R$ and similarly for $N$, and $\operatorname{Ext}_{A}^{i}(M, N)=0$ for all $i>\operatorname{dim} A$.

Proof. We remark again that $A$ is also Gorenstein by [7].

For the second statement, we induce on $d:=\operatorname{dim} A=\operatorname{dim} R+\operatorname{dim} S$. Suppose that $A$ has dimension 0 . In this case duality yields $\operatorname{Ext}_{A}^{i}(M, N)^{*} \simeq$ $\operatorname{Tor}_{i}^{A}\left(M, N^{*}\right)$. Thus it suffices to prove $\operatorname{Tor}_{i}^{A}\left(M, N^{*}\right)=0$ for all $i>0$.

We first claim that $\operatorname{Hom}_{R \otimes_{k} S}\left(M_{R} \otimes_{k} S, R \otimes_{k} S\right) \simeq \operatorname{Hom}_{R}\left(M_{R}, R\right) \otimes_{k} S$. To see this, note that these modules are naturally isomorphic if $M_{R}$ is free $R$-module. In general, let $R^{m} \stackrel{\rho}{\rightarrow} R^{n} \rightarrow M_{R} \rightarrow 0$ be a presentation of $M_{R}$ over $R$. Let $A^{\prime}:=R \otimes_{k} S$ and $M^{\prime}:=M_{R} \otimes_{k} S$. This yields a presentation $\left(A^{\prime}\right)^{m} \rightarrow\left(A^{\prime}\right)^{n} \rightarrow M^{\prime} \rightarrow 0$ of $M^{\prime}$ over $A^{\prime}$. We obtain a commutative diagram

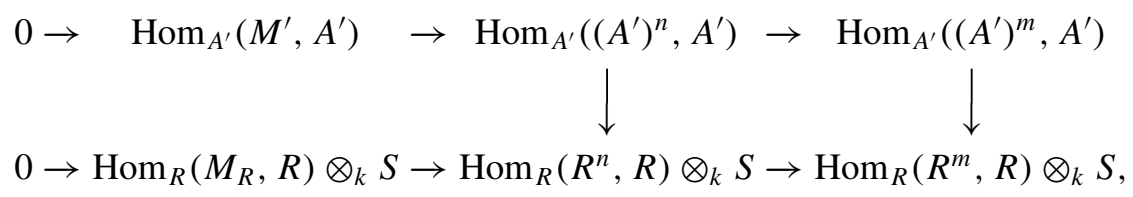$$
0 \rightarrow \operatorname{Hom}_{R}\left(M_{R}, R\right) \otimes_{k} S \rightarrow \operatorname{Hom}_{R}\left(R^{n}, R\right) \otimes_{k} S \rightarrow \operatorname{Hom}_{R}\left(R^{m}, R\right) \otimes_{k} S,
$$

where the first row is exact and the vertical arrows are isomorphisms. To establish the claim we only need to know that the bottom row is exact, but this follows from the fact that $0 \rightarrow \operatorname{Hom}_{R}\left(M_{R}, R\right) \rightarrow \operatorname{Hom}_{R}\left(R^{n}, R\right) \rightarrow$ $\operatorname{Hom}_{R}\left(R^{m}, R\right)$ is an exact sequence of $k$-modules and $S$ is flat as a $k$-module.

Localizing the isomorphism in the claim above at $P$, we see that the $A$ module $M^{*}:=\operatorname{Hom}_{A}(M, A)$ comes from the $R$-module $\operatorname{Hom}_{R}\left(M_{R}, R\right)$. Similarly $N^{*}:=\operatorname{Hom}_{A}(N, A)$ comes from the $S$-module $\operatorname{Hom}_{S}\left(N_{S}, S\right)$. Hence there is no distinction between proving $\operatorname{Tor}_{i}^{A}\left(M, N^{*}\right)=0$ for all $i>0$ and proving $\operatorname{Tor}_{i}^{A}(M, N)=0$ for all $i>0$. We will prove the latter.

Let $(\mathbf{F}, f)$ be an $R$-free resolution of $M_{R}$. Then $\mathbf{F}$ is an exact sequence of $k$ modules, and since $S$ is flat as a $k$-module, $\mathbf{F} \otimes_{k} S$ is an exact sequence, of $R \otimes_{k} S$-modules. Thus $\left(\mathbf{F} \otimes_{k} S\right)_{P}$ is an $A$-free resolution of $M$. To show that $\operatorname{Tor}_{i}^{A}(M, N)=0$ for all $i>0$ we will simply show that the complex $\left(\mathbf{F} \otimes_{k} S\right)_{P} \otimes_{A} N$ is acyclic (meaning the homology is zero except in degree zero). 
For all $i$ we have a commutative diagram

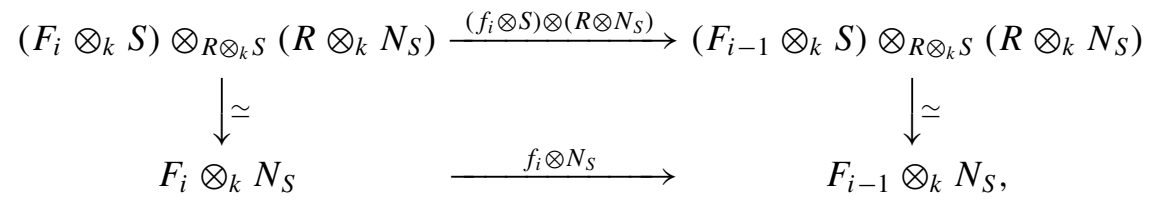

where the vertical arrows are the natural isomorphisms. Hence $\left(\mathbf{F} \otimes_{k} S\right) \otimes_{R} \otimes_{k} S$ $\left(R \otimes_{k} N_{S}\right)$ and $\mathbf{F} \otimes_{k} N_{S}$ are isomorphic complexes of $R \otimes_{k} S$-modules. Since $N_{S}$ is flat as a $k$-module, the latter is acyclic, and therefore so is $\left(\mathbf{F} \otimes_{k} R_{S}\right) \otimes_{R} \otimes_{k} S$ $\left(R \otimes_{k} N_{S}\right)$. Finally, localizing at $P$ we get that $\left(\mathbf{F} \otimes_{k} S\right)_{P} \otimes_{A} N$ is acyclic, and this finishes the proof in the $d=0$ case.

Now without loss of generality assume that $\operatorname{dim} R>0$. From the discussion above we know that $M_{1} \simeq\left(\left(M_{R}\right)_{1} \otimes_{k} S\right)_{P}$. Let $x$ be a non-zerodivisor on both $\left(M_{R}\right)_{1}$ and $R$. Then $x \otimes 1$ is a non-zerodivisor on $M_{1}, A$ and $N$, and we have

$$
\begin{gathered}
A /(x \otimes 1) \simeq\left(R /(x) \otimes_{k} S\right)_{P}, \\
M_{1} /(x \otimes 1) M_{1} \simeq\left(\left(M_{R}\right)_{1} / x\left(M_{R}\right)_{1} \otimes_{k} S\right)_{P}
\end{gathered}
$$

and

$$
N /(x \otimes 1) N \simeq\left(R /(x) \otimes_{k} N_{S}\right)_{P} .
$$

Hence by induction we have that

$$
\operatorname{Ext}_{A /(x \otimes 1)}^{i}\left(M_{1} /(x \otimes 1) M_{1}, N /(x \otimes 1) N\right)=0
$$

for all $i>d-1$. Now (3.3.1) and (3.3.2) show that $\operatorname{Ext}_{A}^{i}\left(M_{1}, N\right)=0$ for all $i>d-1$, which means that $\operatorname{Ext}_{A}^{i}(M, N)=0$ for all $i>d$.

\section{What does the vanishing of Ext mean?}

Many of the results in this section are closely related to the work of Auslander and Bridger. See [1], and the writeup [4] of the contents of [1]. However, none of the results below is explicitly in these works, and we found they gave us a better understanding of what the vanishing of Ext means.

The natural maps $M^{*} \otimes_{R} N \rightarrow \operatorname{Hom}_{R}(M, N)$ and $M \otimes_{R} N^{*} \rightarrow \operatorname{Hom}_{R}(M, N)^{*}$ Assume that $M$ is maximal Cohen-Macaulay. From the short exact sequence $0 \rightarrow M_{1} \rightarrow F \rightarrow M \rightarrow 0$ we get the dual short exact sequence $0 \rightarrow M^{*} \rightarrow$ $F^{*} \rightarrow M_{1}^{*} \rightarrow 0$, and these yield a commutative diagram

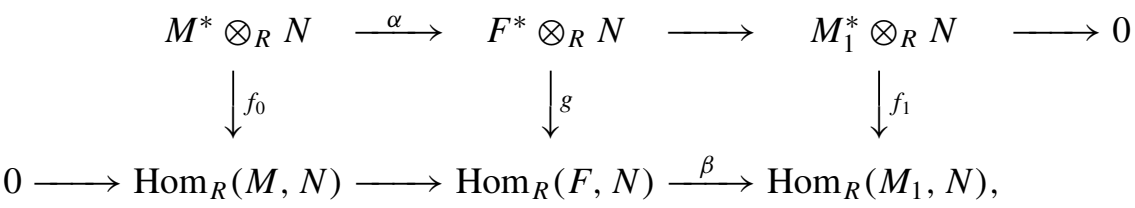


where the vertical arrows are the natural maps $M^{*} \otimes_{R} N \rightarrow \operatorname{Hom}_{R}(M, N)$ given by $\phi \otimes n \mapsto\{m \mapsto \phi(m) n\}$. Note that $g$ is an isomorphism (since $F$ is free), $\operatorname{ker} \alpha \simeq \operatorname{Tor}_{1}^{R}\left(M_{1}^{*}, N\right)$ and coker $\beta \simeq \operatorname{Ext}_{R}^{1}(M, N)$. From this diagram one easily deduces the following three facts.

(1) $\operatorname{ker} f_{1} \simeq \operatorname{coker} f_{0}$,

(2) $\operatorname{Tor}_{1}^{R}\left(M_{1}^{*}, N\right) \simeq \operatorname{ker} f_{0}$, and

(3) $\operatorname{Ext}_{R}^{1}(M, N) \simeq \operatorname{coker} f_{1}$.

Building a diagram as above for each of the exact sequences $0 \rightarrow M_{i+1} \rightarrow$ $F_{i} \rightarrow M_{i} \rightarrow 0$ and using the corresponding three facts as above, we see that we have exact sequences

$$
\begin{aligned}
0 \rightarrow \operatorname{Ext}_{R}^{1}\left(M_{i-2}, N\right) \rightarrow M_{i}^{*} & \otimes_{R} N \\
& \rightarrow \operatorname{Hom}_{R}\left(M_{i}, N\right) \rightarrow \operatorname{Ext}_{R}^{1}\left(M_{i-1}, N\right) \rightarrow 0,
\end{aligned}
$$

and

$$
\begin{aligned}
0 \rightarrow \operatorname{Tor}_{1}^{R}\left(M_{i+1}^{*}, N\right) \rightarrow M_{i}^{*} & \otimes_{R} N \\
& \rightarrow \operatorname{Hom}_{R}\left(M_{i}, N\right) \rightarrow \operatorname{Tor}_{1}^{R}\left(M_{i+2}^{*}, N\right) \rightarrow 0 .
\end{aligned}
$$

For $i \geq 2$ the first exact sequence can be written as

$$
\begin{aligned}
0 \rightarrow \operatorname{Ext}_{R}^{i-1}(M, N) \rightarrow M_{i}^{*} & \otimes_{R} N \\
& \rightarrow \operatorname{Hom}_{R}\left(M_{i}, N\right) \rightarrow \operatorname{Ext}_{R}^{i}(M, N) \rightarrow 0 .
\end{aligned}
$$

An immediate observation is

Proposition 5.2. Let $R$ be a local Gorenstein ring, and let $M$ and $N$ be finitely generated $R$-modules with $M$ maximal Cohen-Macaulay. Then $\operatorname{Ext}_{R}^{i}(M, N)=0$ for all $i \gg 0$ if and only if the natural maps $M_{i}^{*} \otimes_{R} N \rightarrow$ $\operatorname{Hom}_{R}\left(M_{i}, N\right)$ are isomorphisms for all $i \gg 0$.

Note also that building exact sequences (5.1) for arbitrarily large negative syzygies of $M$, and then splicing the resulting exact sequences together, we obtain a doubly infinite long exact sequence

$$
\begin{aligned}
\cdots \rightarrow M_{i}^{*} \otimes_{R} N \rightarrow & \operatorname{Hom}_{R}\left(M_{i}, N\right) \\
& \rightarrow M_{i+i}^{*} \otimes_{R} N \rightarrow \operatorname{Hom}_{R}\left(M_{i+1}, N\right) \rightarrow \cdots .
\end{aligned}
$$

Now suppose that $N$ is maximal Cohen-Macaulay and that $\operatorname{Ext}_{R}^{1}(M, N)=$ 0 . From the short exact sequence $0 \rightarrow M_{1} \rightarrow F \rightarrow M \rightarrow 0$ we get the short 
exact sequence $0 \rightarrow \operatorname{Hom}_{R}(M, N) \rightarrow \operatorname{Hom}_{R}(F, N) \rightarrow \operatorname{Hom}_{R}\left(M_{1}, N\right) \rightarrow$ 0 , and a commutative diagram

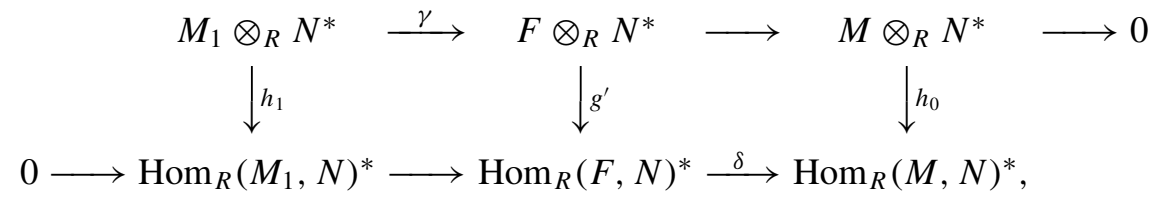

where the vertical arrows are the natural maps $M \otimes_{R} N^{*} \rightarrow \operatorname{Hom}_{R}(M, N)^{*}$ given by $m \otimes \phi \mapsto\{\psi \mapsto \phi(\psi(m))\}$. Note that $g^{\prime}$ is an isomorphism (since $F$ is free), $\operatorname{ker} \gamma \simeq \operatorname{Tor}_{1}^{R}\left(M, N^{*}\right)$ and coker $\delta \simeq \operatorname{Ext}_{R}^{1}\left(\operatorname{Hom}_{R}\left(M_{1}, N\right), R\right)$. Regarding this diagram, we have the following three facts.

(1) $\operatorname{ker} h_{0} \simeq \operatorname{coker} h_{1}$,

(2) $\operatorname{Tor}_{1}^{R}\left(M, N^{*}\right) \simeq \operatorname{ker} h_{1}$, and

(3) $\operatorname{Ext}_{R}^{1}\left(\operatorname{Hom}_{R}\left(M_{1}, N\right), R\right) \simeq \operatorname{coker} h_{0}$.

Now assume that $\operatorname{Ext}_{R}^{i}(M, N)=0$ for all $i>0$, equivalently $\operatorname{Ext}_{R}^{1}\left(M_{i}, N\right)=$ 0 for all $i \geq 0$. Constructing such a diagram as above for each of the short exact sequences $0 \rightarrow M_{i+1} \rightarrow F_{i} \rightarrow M_{i} \rightarrow 0$ and using the corresponding three facts as above we obtain exact sequences

$$
\begin{aligned}
0 \rightarrow \operatorname{Tor}_{i}^{R}\left(M, N^{*}\right) \rightarrow & M_{i} \otimes_{R} N^{*} \\
& \stackrel{h_{i}}{\longrightarrow} \operatorname{Hom}_{R}\left(M_{i}, N\right)^{*} \rightarrow \operatorname{Tor}_{i-1}^{R}\left(M, N^{*}\right) \rightarrow 0
\end{aligned}
$$

for $i \geq 2$. From Theorem 2.1 we know that $\operatorname{Ext}_{R}^{i}(M, N)=0$ for all $i \gg 0$ if and only if $\operatorname{Tor}_{i}^{R}\left(M, N^{*}\right)=0$ for all $i \gg 0$. Hence

Proposition 5.5. Let $R$ be a local Gorenstein ring, and let $M$ and $N$ be finitely generated $R$-modules with $N$ maximal Cohen-Macaulay. Then $\operatorname{Ext}^{i}(M, N)=0$ for all $i \gg 0$ implies the natural maps $M_{i} \otimes_{R} N^{*} \rightarrow$ $\operatorname{Hom}_{R}\left(M_{i}, N\right)^{*}$ are isomorphisms for all $i \gg 0$.

Theorem 5.9 below contains a similar result.

\section{Ext and Stable Hom}

Recall that the stable $\operatorname{Hom}, \operatorname{Hom}_{R}(M, N)$, is the cokernel of the natural map $M^{*} \otimes_{R} N \rightarrow \operatorname{Hom}_{R}(M, N)$. Equivalently, it is the quotient of $\operatorname{Hom}_{R}(M, N)$ by maps $f: M \rightarrow N$ which factor through a free module. Stable Homs offer 
a convenient way of interpreting the vanishing of all higher $\operatorname{Ext}_{R}^{i}(M, N)$ : from (5.1) (and the exact sequence involving Ext preceding it) we see that

$$
\operatorname{Ext}_{R}^{i}(M, N) \simeq \underline{\operatorname{Hom}}_{R}\left(M_{i}, N\right)
$$

for $i \geq 1$. Hence we may record the following as a corollary of 5.2.

Corollary 5.7. Let $R$ be a local Gorenstein ring, and let $M$ and $N$ be finitely generated $R$-modules with $M$ maximal Cohen-Macaulay. Then $\operatorname{Ext}_{R}^{i}(M, N)=0$ for all $i \gg 0$ if and only if for all $i \gg 0$ every map $M_{i} \rightarrow N$ factors through a free module.

The next Proposition allows us to shift among the stable Homs with ease, which often can clarify basic vanishing results concerning Ext. (cf. Remark 2.2 and (1.2).)

Proposition 5.8. Let $M$ and $N$ be finitely generated maximal CohenMacaulay modules over the local Gorenstein ring $R$. Then

(1) $\underline{\operatorname{Hom}}_{R}(M, N) \simeq \underline{\operatorname{Hom}}_{R}\left(N^{*}, M^{*}\right)$.

(2) $\underline{\operatorname{Hom}}_{R}(M, N) \simeq \underline{\operatorname{Hom}}_{R}\left(M_{t}, N_{t}\right)$ for all $t \in \mathrm{Z}$.

Proof. (1). The isomorphism is induced by the obvious mapping $\operatorname{Hom}_{R}(, R): \operatorname{Hom}_{R}(M, N) \rightarrow \operatorname{Hom}_{R}\left(N^{*}, M^{*}\right)$. The fact that the induced map on stable Homs is an isomorphism is straightforward (since $M$ and $N$ are reflexive) provided it is well-defined. But this is clear since if $f \in$ $\operatorname{Hom}_{R}(M, N)$ factors through a free module $F$, then $\operatorname{Hom}_{R}(f, R)$ factors through $F^{*}$.

(2). It is enough to prove (2) in the case $t=1$. Given a map $f \in \operatorname{Hom}_{R}(M, N)$ we get a map $f_{1} \in \operatorname{Hom}_{R}\left(M_{1}, N_{1}\right)$ by completing the diagram

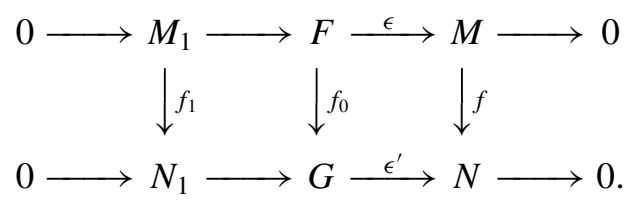

Define $\Phi: \underline{\operatorname{Hom}}_{R}(M, N) \rightarrow \underline{\operatorname{Hom}}_{R}\left(M_{1}, N_{1}\right)$ by $\Phi(\bar{f})=\bar{f}_{1}$.

We first show that $\Phi(\bar{f})$ is determined independent of the choice of chain map $\left\{f_{0}, f_{1}\right\}$. Suppose that $g_{0}: F \rightarrow G$ and $g_{1}: M_{1} \rightarrow N_{1}$ are two other maps making the diagram (5.8.1) commute and such that $\epsilon^{\prime} g_{0}=f \epsilon$. Then we have the standard homotopy $h: F \rightarrow N_{1}$ such that $f_{1}-g_{1}=h\left(M_{1} \hookrightarrow F\right)$. That is, $f_{1}-g_{1}$ factors through a free module, so that $\bar{f}_{1}=\bar{g}_{1}$ in $\underline{\operatorname{Hom}}_{R}\left(M_{1}, N_{1}\right)$. 
Next we show $\Phi$ is well-defined. Suppose that $f \in \operatorname{Hom}_{R}(M, N)$ factors through a free module $H$. Then $f_{1}=0$ completes the diagram

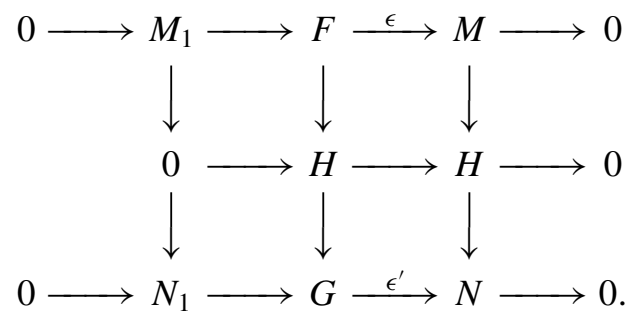

Hence, $\Phi(\bar{f})=0$, as desired.

In order to show that $\Phi$ is an isomorphism we exhibit its inverse. Let $g$ be in $\operatorname{Hom}_{R}\left(M_{1}, N_{1}\right)$. We dualize and complete the diagram

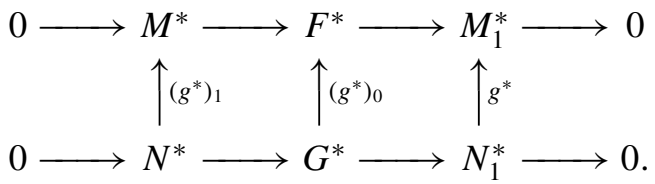

Define $\Psi: \underline{\operatorname{Hom}}_{R}\left(M_{1}, N_{1}\right) \rightarrow \underline{\operatorname{Hom}}_{R}(M, N)$ by $\Psi(\bar{g})=\overline{\left(g^{*}\right)_{1}^{*}}$. It's not hard to see that $\Phi$ and $\Psi$ are inverses of one another (since $M$ and $N$ are reflexive).

\section{Vanishing Ext and Cohen-Macaulayness}

We end with a theorem which shows the relationship between the vanishing of $d$ consecutive Ext modules and the Cohen-Macaulayness of a certain tensor product.

Theorem 5.9. Let $R$ be a d-dimensional local Gorenstein ring, and let $M$ and $N$ be maximal Cohen-Macaulay modules. Consider the following two conditions.

(1) $M^{*} \otimes_{R} N$ is maximal Cohen-Macaulay,

(2) $\operatorname{Ext}_{R}^{1}(N, M)=\cdots=\operatorname{Ext}_{R}^{d}(N, M)=0$.

Then (2) implies (1). If we assume that $\operatorname{Ext}_{R}^{1}(N, M), \ldots, \operatorname{Ext}_{R}^{d}(N, M)$ have finite length, then (1) implies (2). Furthermore, if (1) holds then $\operatorname{Hom}_{R}(N, M)$ is maximal Cohen-Macaulay and $M^{*} \otimes_{R} N \simeq \operatorname{Hom}_{R}(N, M)^{*}$. If (1) holds and $R$ is also integrally closed, then $M^{*} \otimes_{R} N \simeq \operatorname{Hom}_{R}(M, N)$.

Proof. We first prove that if (1) holds then the $R$-module $\operatorname{Hom}_{R}(N, M)$ is maximal Cohen-Macaulay and $M^{*} \otimes_{R} N \simeq \operatorname{Hom}_{R}(N, M)^{*}$. Note that as both $M$ and $N$ are reflexive, the natural map $\operatorname{Hom}_{R}(N, M) \rightarrow \operatorname{Hom}_{R}\left(M^{*}, N^{*}\right)$ is an 
isomorphism. Since $M^{*} \otimes_{R} N$ is maximal Cohen-Macaulay so is $\left(M^{*} \otimes_{R} N\right)^{*}$, and we have

$$
\begin{aligned}
\left(M^{*} \otimes_{R} N\right)^{*} & =\operatorname{Hom}_{R}\left(M^{*} \otimes_{R} N, R\right) \\
& \simeq \operatorname{Hom}_{R}\left(M^{*}, N^{*}\right) \quad \text { by Hom-tensor adjointness } \\
& \simeq \operatorname{Hom}_{R}(N, M)
\end{aligned}
$$

Hence $\operatorname{Hom}_{R}(N, M)$ is maximal Cohen-Macaulay and $M^{*} \otimes_{R} N \simeq\left(M^{*} \otimes_{R}\right.$ $N)^{* *} \simeq \operatorname{Hom}_{R}(N, M)^{*}$.

We prove $(1) \Longrightarrow(2)$ under the assumption that $\operatorname{Ext}_{R}^{1}(N, M), \ldots, \operatorname{Ext}_{R}^{d}(N$, $M$ ) have finite length. We induce on $d$. The case in which $d=0$ is vacuous.

$d=1$. Since $\operatorname{Ext}_{R}^{1}(N, M)$ has finite length and $M$ and $N$ are maximal Cohen-Macaulay, we can choose a non-zerodivisor $x \in R$ such that $x$ is a non-zerodivisor on both $M$ and $N$ and $x \operatorname{Ext}_{R}^{1}(N, M)=0$. For any $R$ module $X$ we let $\bar{X}$ denote $X / x X$, and we let * indicate Hom into either $R$ or $\bar{R}$ depending on the module in question. We have the short exact sequence $0 \rightarrow M \stackrel{x}{\rightarrow} M \rightarrow \bar{M} \rightarrow 0$, which yields the exact sequence

$$
\begin{aligned}
0 \rightarrow \operatorname{Hom}_{R}(N, M) \stackrel{x}{\rightarrow} \operatorname{Hom}_{R}(N, M) & \\
& \rightarrow \operatorname{Hom}_{\bar{R}}(\bar{N}, \bar{M}) \rightarrow \operatorname{Ext}_{R}^{1}(N, M) \rightarrow 0 .
\end{aligned}
$$

Hence length $\left(\operatorname{Hom}_{\bar{R}}(\bar{N}, \bar{M})\right)=$ length $\left(\overline{\operatorname{Hom}_{R}(N, M)}\right)+$ length $\left(\operatorname{Ext}_{R}^{1}(N, M)\right)$. Note that for any maximal Cohen-Macaulay $R$-module $X, \overline{X^{*}} \simeq \bar{X}^{*}$. We have

$$
\begin{array}{rlrl}
\text { length }\left(\operatorname{Hom}_{\bar{R}}(\bar{N}, \bar{M})\right) & =\text { length }\left(\left(\bar{M}^{*} \otimes_{\bar{R}} \bar{N}\right)^{*}\right) & & \text { by (5.9.1) } \\
& =\text { length }\left(\bar{M}^{*} \otimes_{\bar{R}} \bar{N}\right) & & \text { since } \bar{R} \text { is 0-dimensional } \\
& =\text { length }\left(\overline{M^{*}} \otimes_{\bar{R}} \bar{N}\right) & & \text { since } \bar{M}^{*} \simeq \overline{M^{*}} \\
& =\text { length }\left(\overline{M^{*} \otimes_{R} N}\right) & \\
& =\text { length }\left(\overline{\operatorname{Hom}_{R}(N, M)^{*}}\right) & & \text { by (5.9.1) } \\
& =\text { length }\left(\overline{\operatorname{Hom}_{R}(N, M)}\right) & \\
& =\text { length }\left(\overline{\operatorname{Hom}_{R}(N, M)}\right) & \text { since } \bar{R} \text { is 0-dimensional. }
\end{array}
$$

Therefore

length $\left(\overline{\operatorname{Hom}_{R}(N, M)}\right)=$ length $\left(\overline{\operatorname{Hom}_{R}(N, M)}\right)+$ length $\left(\operatorname{Ext}_{R}^{1}(N, M)\right)$, and so $\operatorname{Ext}_{R}^{1}(N, M)=0$.

$d>1$. Choose a parameter $x \in \cap_{i=1}^{d} \operatorname{ann}_{R} \operatorname{Ext}_{R}^{i}(N, M)$. We have $\bar{M}^{*} \otimes_{\bar{R}}$ $\bar{N} \simeq \overline{M^{*} \otimes_{R} N}$ is maximal Cohen-Macaulay. The short exact sequence $0 \rightarrow$ 
$M \stackrel{x}{\rightarrow} M \rightarrow \bar{M} \rightarrow 0$ yields exact sequences

$$
0 \rightarrow \operatorname{Ext}_{R}^{i}(N, M) \rightarrow \operatorname{Ext}_{R}^{i}(N, \bar{M}) \rightarrow \operatorname{Ext}_{R}^{i+1}(N, M) \rightarrow 0
$$

for $i=1, \ldots, d-1$. Hence $\operatorname{Ext}_{R}^{i}(N, \bar{M})$ have finite length for $1 \leq i \leq d-1$. Since $\operatorname{Ext}_{R}^{i}(N, \bar{M}) \simeq \operatorname{Ext}_{\bar{R}}^{i}(\bar{N}, \bar{M})$ for all $i$ [5], it follows by induction that $\left.\operatorname{Ext}_{\bar{R}}(\bar{N}, \bar{M})=\cdots=\operatorname{Ext}_{\bar{R}}^{\frac{d-1}{(N}}, \bar{M}\right)=0$. Now the exact sequences (5.9.2), for $i=1, \ldots, d-1$, and the fact that $\operatorname{Ext}_{R}^{i}(N, \bar{M}) \simeq \operatorname{Ext}_{\bar{R}}^{i}(\bar{N}, \bar{M})$ for all $i \geq 1$ gives $\operatorname{Ext}_{R}^{i}(N, M)=0$ for $i=1, \ldots, d$.

Assume (2). Note then that $\operatorname{Hom}_{R}(N, M)$ is maximal Cohen-Macaulay: let $\mathbf{F}: \cdots \rightarrow F_{1} \rightarrow F_{0} \rightarrow N \rightarrow 0$ be an $R$-free resolution of $N$. Applying $\operatorname{Hom}_{R}(, M)$ and using our hypothesis we get the exact sequence

$$
0 \rightarrow \operatorname{Hom}_{R}(N, M) \rightarrow \operatorname{Hom}_{R}\left(F_{0}, M\right) \rightarrow \cdots \rightarrow \operatorname{Hom}_{R}\left(F_{d+1}, M\right) .
$$

Each $\operatorname{Hom}_{R}\left(F_{i}, M\right)$ is maximal Cohen-Macaulay since $M$ is. By counting depths along this exact sequence we get the desired conclusion.

For (2) $\Longrightarrow(1)$ we again induce on $d$. The case $d=0$ is trivial.

$d=1$. Let $x$ be a non-zerodivisor on both $M$ and $N$. The short exact sequence $0 \rightarrow M \stackrel{x}{\rightarrow} M \rightarrow \bar{M} \rightarrow 0$ and our hypothesis yield the short exact sequence

$$
0 \rightarrow \operatorname{Hom}_{R}(N, M) \stackrel{x}{\rightarrow} \operatorname{Hom}_{R}(N, M) \rightarrow \operatorname{Hom}_{\bar{R}}(\bar{N}, \bar{M}) \rightarrow 0 .
$$

Therefore

$$
\overline{\operatorname{Hom}_{R}(N, M)} \simeq \operatorname{Hom}_{\bar{R}}(\bar{N}, \bar{M})
$$

Consider the natural map $M^{*} \otimes_{R} N \stackrel{h}{\rightarrow} \operatorname{Hom}_{R}(N, M)^{*}$. We have a commutative diagram

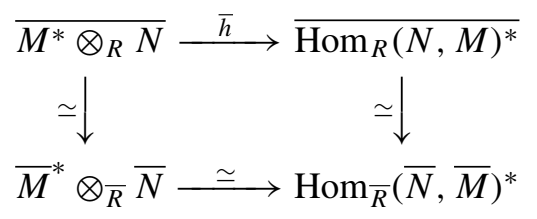

where the right vertical arrow comes from (5.9.3) and the bottom arrow is the isomorphism of (5.9.1). Thus $h$ is an isomorphism modulo $x$. By Nakayama's lemma, $h$ must be onto. Now we have a short exact sequence

$$
0 \rightarrow K \rightarrow M^{*} \otimes_{R} N \stackrel{h}{\rightarrow} \operatorname{Hom}_{R}(N, M)^{*} \rightarrow 0 .
$$


The fact that $\operatorname{Hom}_{R}(N, M)$ is maximal Cohen-Macaulay implies that $\bar{K}=0$, and therefore $K=0$. Thus $h$ is an isomorphism and $M^{*} \otimes_{R} N$ is maximal Cohen-Macaulay.

$d>1$. For $x$ a non-zerodivisor on $M$ and $N$, the hypothesis yields $\operatorname{Ext}_{\bar{R}}(\bar{N}, \bar{M})=\cdots=\operatorname{Ext}_{\bar{R}}^{d-1}(\bar{N}, \bar{M})=0$, so by induction $\bar{M}^{*} \otimes_{\bar{R}} \bar{N}$ is maximal Cohen-Macaulay, which means so is $M^{*} \otimes_{R} N$.

Finally suppose that $R$ is integrally closed. There is always a natural map from $\operatorname{Hom}_{R}(M, N) \rightarrow \operatorname{Hom}_{R}(N, M)^{*}$ obtained by composition, and this map is an isomorphism if either $N$ or $M$ is free. Since $M$ and $N$ are maximal CohenMacaulay modules and $R_{P}$ is regular if the height of $P$ is at most one, it follows that this natural map is an isomorphism in codimension one. It is a standard result that a map between reflexive modules which is an isomorphism in codimension one must itself be an isomorphism. Hence, as both $\operatorname{Hom}_{R}(M, N)$ and $\operatorname{Hom}_{R}(N, M)^{*}$ are reflexive, the natural map $\operatorname{Hom}_{R}(M, N) \rightarrow \operatorname{Hom}_{R}(N, M)^{*}$ is an isomorphism. The stated isomorphism of $M^{*} \otimes_{R} N$ with $\operatorname{Hom}_{R}(M, N)$ follows from the above paragraph.

\section{Questions}

This work leaves quite a few questions unresolved. We list a few for further study. Perhaps the most intriguing is

1. Are all local Gorenstein rings $\mathrm{AB}$ rings?

Some other interesting questions are:

2. Let $R$ and $S$ be AB rings which are essentially of finite type over the same field $k$. Is $R \otimes_{k} S$ locally an AB ring if neither $R$ nor $S$ is a complete intersection?

3. Are localizations of $\mathrm{AB}$ rings $\mathrm{AB}$ rings?

4. Are $\mathrm{AB}$ rings Ext-bounded?

NOTE ADDED IN PROOF. This question has recently been answered in the negative by the second author and L. M. Şeqa in 'Nonvanishing cohomology and classes of Gorenstein rings', Preprint, available at http://www.arxiv.org/abs/math/0306001.

\section{REFERENCES}

1. Auslander, M., and Bridger, M., Stable Module Theory, Mem. Amer. Math. Soc. 94 (1969).

2. Avramov, L. L., and Buchweitz, R-.O., Support varieties and cohomology over complete intersections, Invent. Math. 142 (2000), 285-318.

3. Lescot, J., Asymptotic properties of Betti numbers of modules over certain rings, J. Pure Appl. Algebra 38 (1985), 287-298. 
4. Maşek, V., Gorenstein dimension and torsion of modules over commutative Noetherian rings, Comm. Algebra (2000), 5783-5812.

5. Rees, D., A theorem of homological algebra, Proc. Cambridge Philos. Soc. 52 (1956), 605610.

6. Rotman, J., An Introduction to Homological Algebra, Academic Press, New York, 1979.

7. Watanabe, K. I., Ishikawa, T., Tachibana, S., and Otsuka, K., On tensor products of Gorenstein rings, J. Math. Kyoto Univ. 9 (1969), 413-423.

DEPARTMENT OF MATHEMATICS

UNIVERSITY OF KANSAS

LAWRENCE, KS 66045

USA

E-mail: huneke@math.ukans.edu
DEPARTMENT OF MATHEMATICS

UNIVERSITY OF TEXAS AT ARLINGTON

ARLINGTON, TX 76019

USA

E-mail: djorgens@math.uta.edu 